\title{
PARA ALÉM DA COMPUTAÇÃO ESTATÍSTICA: O USO DO AMBIENTE R PARA O ENSINO DE MÉTODOS NUMÉRICOS
}

\author{
Elisa Henning - DMAT/UDESC - elisa.henning@udesc.br \\ Marcelo Savio Ramos - UDESC - marcelo.savio.ramos@gmail.com \\ Rogério de Aguiar - DMAT /UDESC - rogerio.aguiar@udesc.br \\ Ivanete Zuchi Siple - DMAT/UDESC - ivanete.siple@udesc.br \\ Luciane Mulazani dos Santos - DMAT /UDESC - luciane.mulazani@udesc.br
}

\begin{abstract}
Resumo: Este trabalho tem por objetivo mostrar algumas potencialidades do GNU $R$ para o ensino de Métodos Numéricos, nomeadamente Zeros de Funções. A partir de uma pesquisa exploratória foram investigadas funções e pacotes existentes no $R$, que poderiam auxiliar nos processos de ensino e aprendizagem destes métodos. Foram também desenvolvidas rotinas com a finalidade de avaliar a complexidade de construção e operacionalização das mesmas, sob a ótica de um aluno de graduação. Os resultados alcançados mostram que o $R$ se constitui em uma alternativa viável de ferramenta computacional para o ensino de métodos numéricos. Como pontos positivos a destacar estão a sua flexibilidade como ambiente computacional, sendo multiplataforma, e a possibilidade de utilização em recursos digitais móveis.
\end{abstract}

Palavras-chave: Ambiente R; Zeros de Funções; Ensino de Métodos Numéricos.

\section{BEYOND STATISTICAL COMPUTING: USING THE R ENVIRONMENT FOR TEACHING NUMERICAL METHODS}

\begin{abstract}
This paper will show some possibilities of using GNU $R$ for teaching numerical methods, namely the zero of a function. Based on exploratory research, existing functions and packages in $R$ that could help in the process of teaching and learning of these methods were investigated. Routines were also developed in order to assess the complexity of their construction and operation. The results achieved show that $R$ is a viable alternative computational tool for teaching numerical methods. Among the positive points to note are its flexibility as a multiplatform computing environment, and the possibility to use it on mobile computing devices.
\end{abstract}

Key-Words: R Environment; Zero of a function; Teaching of Numerical Methods.

\section{Introdução}

De uma maneira geral, podemos dizer que estudar métodos numéricos significa estudar ferramentas matemáticas que nos ajudem na busca por valores aproximados (soluções numéricas) que podem solucionar problemas práticos. Quando estudantes de graduação e de pós-graduação, em seus cursos, se deparam com disciplinas que têm em seus currículos conteúdos de métodos numéricos, têm a possibilidade de construírem conhecimento nesta que é uma área bastante importante para o desenvolvimento de habilidades que permitam a solução de problemas de engenharia, estatística, física, biologia, economia, ciências ambientais, além, é claro, de matemática. Um exemplo é a 
disciplina Cálculo Numérico, na qual o objetivo é o estudo de métodos numéricos para a resolução de problemas em geral aliando custo com precisão durante sua aplicação (AMARAL et al, 2013).

Burden e Faires (2008) nos explicam que uma solução numérica é um valor numérico aproximado que resolve um problema matemático. Buscar uma solução numérica é uma interessante alternativa para solucionar problemas para os quais não há respostas exatas - ou sua obtenção é muito complicada - que podem ser representadas na forma de uma expressão matemática associada às variáveis do problema, o que significa dizer resolver problemas para os quais não existem soluções analíticas.

O estudo de métodos numéricos envolve a aprendizagem de diferentes técnicas de busca de soluções para os problemas matemáticos. Dentre elas, podemos citar: cálculo aproximado de raízes de equações algébricas e transcendentes, solução de sistemas de equações lineares, cálculo de derivadas e integrais e solução de equações diferenciais. A aprendizagem de tantos procedimentos, técnicas e aplicações tão variadas se vê beneficiada quando a tecnologia é utilizada para a realização de cálculos e interpretação das soluções. Um dos objetivos do Cálculo Numérico é o estudo de métodos numéricos para a resolução de problemas em geral aliando custo com precisão durante sua aplicação. (AMARAL et al, 2013)

Percebemos, assim, que nesse contexto do trabalho com métodos numéricos, a tecnologia assume um papel fundamental para auxiliar os estudantes no aprendizado do conteúdo (RAMOS; ARAÚJO, OLIVEIRA, 2008) e os professores no ensino das diferentes técnicas. Estabelece-se, assim, uma estreita ligação entre a Matemática e a tecnologia na forma de ferramentas computacionais, as quais podem ser entendidas como agentes de um processo interativo de ensino e aprendizagem que coloca os alunos como sujeitos participantes efetivos e autônomos no trabalho teórico-prático com soluções numéricas (AMARAL et al, 2013; BORBA; PENTEADO, 2003).

De acordo com Denardi et al (2001), a inserção da tecnologia nestas disciplinas deve permear uma "informatização construcionista" que permita a refl exão e a construção de ideias a partir da relação entre professor, computador, aluno e conhecimento. Deve-se compreender, por exemplo, que o computador não é 0 elemento principal dos processos de ensino e aprendizagem, mas sim uma importante ferramenta auxiliar.

No século $X X$, as técnicas numéricas estudadas pelos alunos nas disciplinas curriculares de métodos numéricos eram implementadas com dificuldade devido aos recursos computacionais então disponíveis. Já na contemporaneidade, graças ao acesso a computadores mais rápidos com grande capacidade para cálculos, podem ser desenvolvidos novos métodos mais modernos e eficientes, além do aprimoramento contínuo dos existentes (AMARAL et al, 2013). A implementação de várias técnicas numéricas se modernizou, tornando mais simples os algoritmos e, consequentemente, os códigos (RAMOS; ARAÚJO, OLIVEIRA, 2008).

Atualmente, é possível resolver problemas matemáticos de forma mais fácil se comparada à utilizada com linguagens de programação como PASCAL, FORTRAN ou C. Pacotes como o Matlab, Scilab, Octave, Freemat e R, graças à grande variedade de funções, têm a capacidade de realizar cálculos relacionados à álgebra linear, processamentos de sinais, construção de gráficos em duas e três dimensões, entre outros, oferecendo uma nova perspectiva para o ensino de Matemática e, em específico, o ensino de Cálculo Numérico (AMARAL et al, 2013). Ressalta-se que o Scilab, Octave, Freemat e R são gratuitos.

Neste artigo, escolhemos discutir a utilização do Ambiente $\mathrm{R}$ no estudo de métodos numéricos, como forma de evidenciar como a tecnologia deste software pode 
ser utilizada de forma bastante útil e eficaz na solução de problemas matemáticos que demandam soluções numéricas. Além disso, queremos mostrar como um ambiente que foi concebido voltado à computação estatística - o $\mathrm{R}$ - também pode ser utilizado no trabalho com métodos numéricos.

\section{0 ambiente $\mathbf{R}$}

O projeto GNU R (R CORE TEAM, 2015), mais conhecido como R, é uma linguagem de programação e também um ambiente para computação estatística e gráfi ca. Gratuito e com código fonte disponível sob a licença GNU GPL, é bastante utilizada por estatísticos e analistas de dados como apoio na resolução de problemas nestas áreas. É também ferramenta importante no ensino de conteúdos de estatística e de probabilidade em cursos de graduação e pós-graduação.

O R Está disponível para download na Internet e pode ser instalado nos sistemas operacionais Windows, Linux e Mac. Além da instalação padrão, diversos pacotes estendem o $\mathrm{R}$ acrescentando-lhe diversas funcionalidades. A sintaxe do $\mathrm{R}$ é similar às linguagens de programação $\mathrm{C}$ e $\mathrm{C}++$, tendo como características o manuseio e tratamento de objetos por meio de funções (rotinas), uso de bibliotecas de funções predefinidas e de programação orientada ao objeto (FERREIRA; CYMROT, 2007).

A interface padrão é o RGui, representada na Figura 1. Percebe-se, por essa representação, que é necessário que o usuário conheça alguns elementos de programação para utilizar o ambiente. Há também outras interfaces, tais como o Rcmdr (FOX, 2005) e o RStudio (RACINE, 2012), que foram desenvolvidas de modo a tornar mais amigáveis algumas funcionalidades, como o carregamento de dados externos, visualização de funções, resultados e gráficos, além da geração de documentos em editores de texto. Destacamos o RStudio, um ambiente de desenvolvimento integrado (IDE - do inglês Integrated Development Environment) para o R, que reúne diversas funcionalidades e ferramentas, além de apresentar duas opções: pode rodar como um aplicativo desktop padrão ou ser acessado por meio de um navegador web.

Figura 1 - Interface RGui

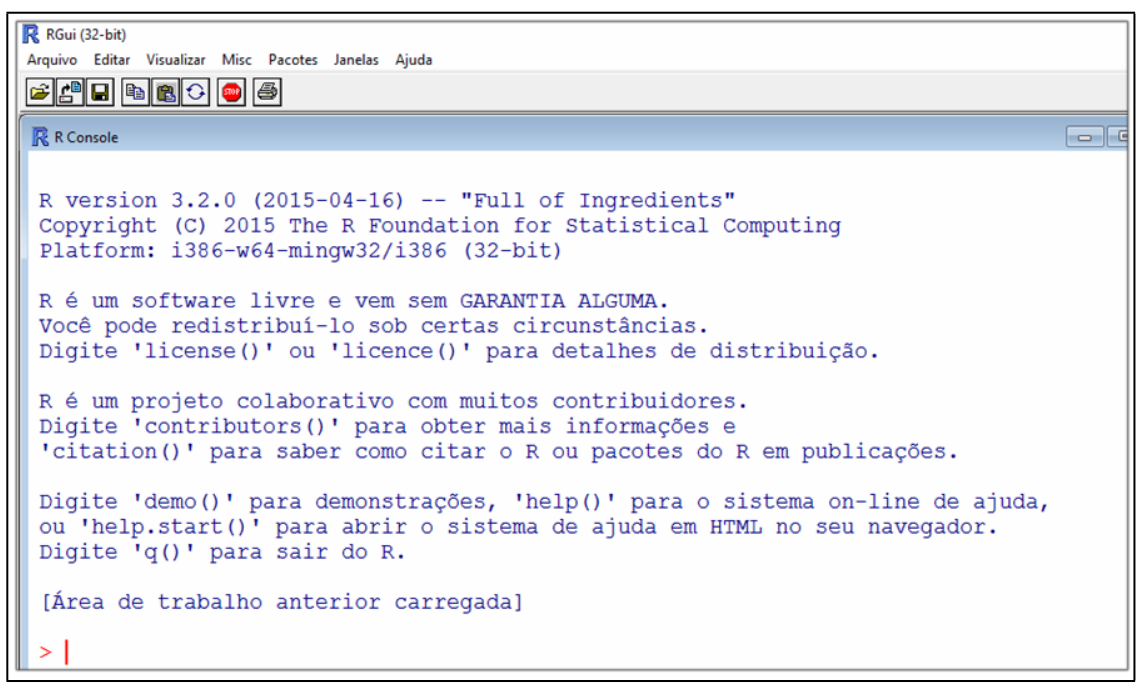

Fonte: produção dos autores

Atualmente, é possível executar o $\mathrm{R}$ também em alguns dispositivos móveis, como smartphones e tablets, porém ainda com algumas restrições. Há versões disponíveis para os sistemas operacionais Android e IOs (Figura 2). 


\section{Figura 2 - Tela do RStudio no sistema IOs}

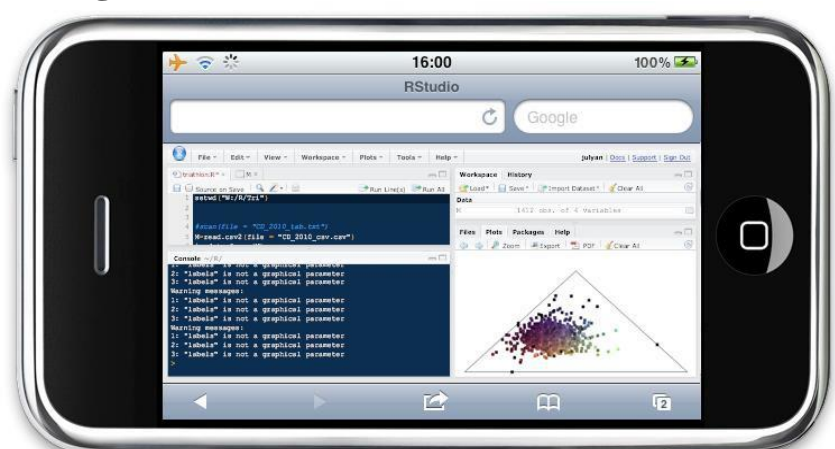

Fonte: http://www.r-bloggers.com/running-r-on-an-iphoneipad-with-rstudio/

\section{Métodos numéricos com o uso do R: zeros de funções}

O R, ainda que seja um ambiente de computação estatística, possui pacotes com funcionalidades que permitem a implementação dos principais métodos numéricos que são estudados em cursos de graduação do Ensino Superior. Entre estes pacotes, estão o pracma (BORCHERS, 2015), o spuRs (OWEN et al., 2014) e o rootSolve (SOETAERT, 2009). Apresentaremos aqui exemplos de práticas realizadas com tais pacotes do $\mathrm{R}$ para encontrar zeros de funções. O zero de uma função é um ponto do domínio da função onde a função se anula, ou seja, um ponto $x_{0}$ é zero da função $f$ se $f\left(x_{0}\right)=0$. Se $x_{0}$ é zero da função $f$ então $x_{0}$ é uma raiz da equação $f(x)=0$, e vice versa.

O pacote pracma oferece implementações de métodos clássicos (bissecção, posição falsa, ponto fixo, Newton-Raphson, secante) e também de métodos mais avançados em análise numérica. Para encontrar raízes de equações (zeros de funções), apresenta funções dos métodos de Newton-Raphson e Secante (que são métodos iterativos para determinação do ponto fixo de uma função). Os argumentos de entrada correspondem à função e ao(s) valor(es) inicial(is), sendo possível controlar também o número de iterações e a tolerância desejada. Como saída, temos os valores aproximados das raízes procuradas da função naquele ponto, além da precisão e do número de iterações executadas.

Na figura 3, pode ser vista a tela de saída do pacote pracma para um exemplo de determinação de zeros da função $f(x)=4 x-\operatorname{sen}(x)$, utilizando-se o método de Newton-Raphson, com valor inicial $x_{0}=0,3$, número de iterações iguais a quatro, obtendo-se por esse método o valor de 0,37055581 para o zero da função.

Figura 3 - Exemplo com as funções newtonRaphson do pacote pracma na interface RStudio

\begin{tabular}{|l||}
\hline Console $/ 2$ \\
$>$ library(pracma) \\
$>$ fx=function $(x) 4 * \sin (x)-\exp (x)$ \\
$>$ newtonkaphson $(\mathrm{fx}, 0.5)$ \\
Sroot \\
{$[1] 0.3705581$} \\
Sf.root \\
{$[1] 0$} \\
Sniter \\
{$[1] 4$} \\
Sestim.prec \\
{$[1] 1.220853 \mathrm{e}-08$} \\
\hline
\end{tabular}

Fonte: produção dos autores. 
O pacote spuRs (OWEN et al., 2014) possui scripts, funções e conjuntos de dados para o livro "Introduction to Scientifi c Programming and Simulation Using R", dos mesmos autores. Os métodos da bisseção (função bisection), ponto fixo (função fixedpoint) e Newton-Raphson são contemplados neste pacote. Esse pacote possui funções que possibilitam ao aluno compreender, por meio de um modo diferente de visualização como ocorre este processo iterativo de convergência, para os métodos da Bissecção e Ponto Fixo. De modo geral, os argumentos de entrada contemplam a função, valores iniciais e tolerância desejada.

A Figura 4 ilustra o processo iterativo para determinar o zero da função $f(x)=\log (x)-x+2$, usando o método da Bissecção e a figura 5 ilustram a representação numérica e gráfica, respectivamente, do método do Ponto Fixo para a solução do zero dessa função. Para o método do Ponto Fixo, há necessidade de declarar uma função $g(x)$ para o processo iterativo. Assim, para determinar o zero da função $f(x)=\log (x)-x+2$ foram necessários entrar com os seguintes dados: função $f(x)=\log (x)-x+2$, função $g(x)=\log (x)+2$, ponto inicial $\mathrm{x}_{0}=3.0$, tolerância $\mathrm{e}=10^{-3}$. A função fixedpoint_show auxilia na visualização do processo de convergência para a raiz.

Figura 4 - Exemplo com a função bisection do pacote spuRs

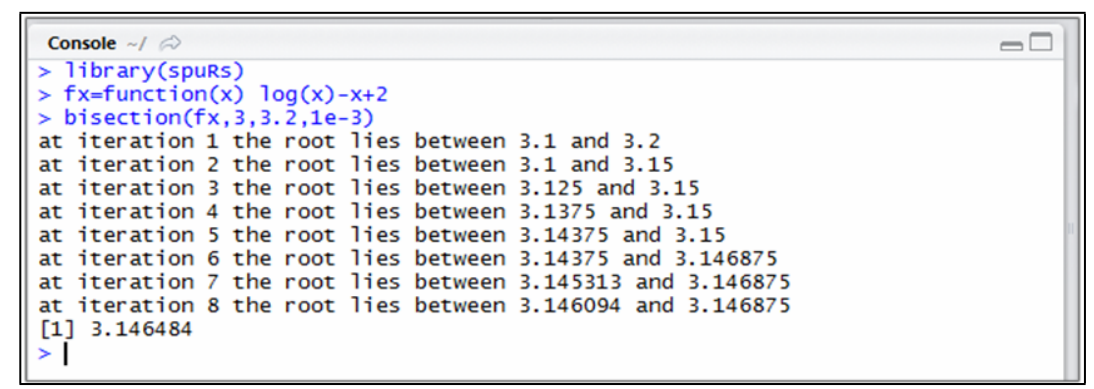

Fonte: produção dos autores

Figura 5 - Exemplo com a função fixedpoint e fixedpoint_show do pacote spuRs

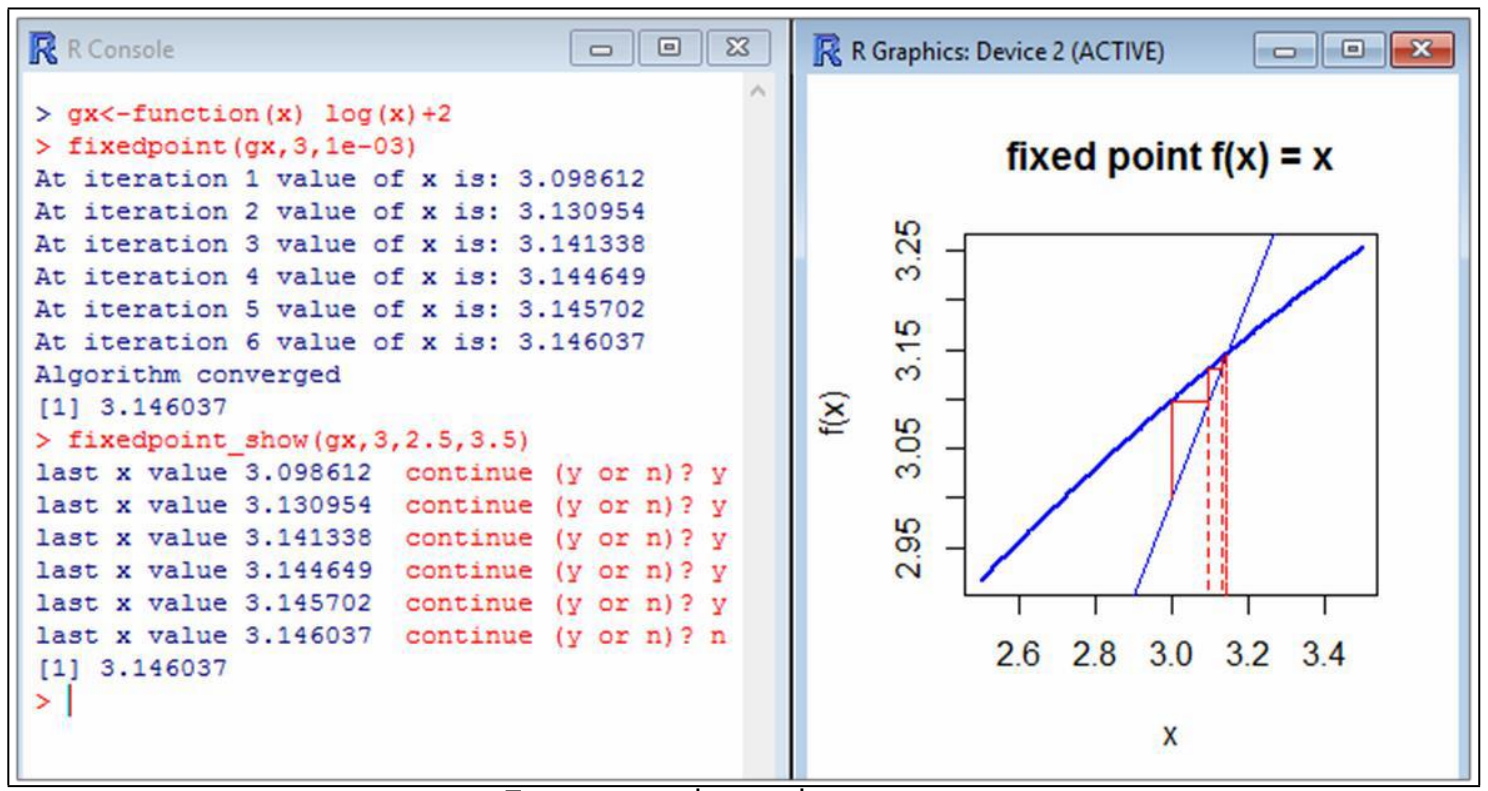

Fonte: produção dos autores 
O pacote rootSolve, de acordo com Soetart (2009) serve a vários propósitos como, por exemplo, para encontrar a raiz de funções não-lineares. Inclui também funções para encontrar o estado estacionário de sistemas de equações diferenciais ordinárias (EDO) e equações diferenciais parciais (EDP), entre outras. Este pacote também inclui algoritmos para localização de raízes únicas e múltiplas $A$ função uniroot(), por exemplo, é utilizada para encontrar uma raiz de uma equação. Já a função uniroot.all(), extensão da função uniroot(), extrai todas as raízes em um dado intervalo, como no exemplo da função $f(x)=\cos (2 x)^{3}$ (Figura 6).

Figura 6 - Exemplo da função uniroot.all do pacote rootSolve

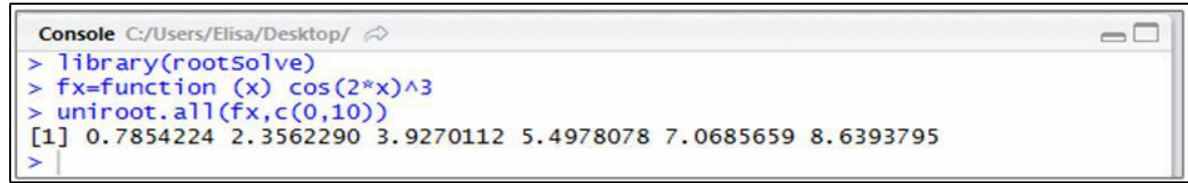

Fonte: produção dos autores

Outra potencialidade da ferramenta é o pacote animation (XIE, 2013). Inicialmente ele foi desenvolvido com a fi nalidade de possibilitar a visualização dinâmica de conceitos estatísticos. Posteriormente, foi estendido para ser uma coleção mais abrangente de ferramentas para gerar animações, assim se tornando uma ferramenta potencial e pode ser útil para a visualização geométrica de alguns métodos numéricos. Com o pacote animation, é possível compreender o conceito de alguns métodos numéricos, por meio da representação passo a passo, permitindo um melhor entendimento da convergência a partir das animações, condição possibilitada pelo uso do software, em oposição ao que poderia ser realizado apenas no ambiente lápis/papel ou quadro/giz.

Dois exemplos presentes no pacote são referentes aos métodos da Bisseção e de Newton-Raphson. Na Figura 7, pode ser visto o comando para a geração da animação que mostra a convergência para a raiz $x=2$ da equação $x^{2}-4=0$. Este é o exemplo que é disponibilizado nesta interface. Para gerar animações com outras funções, basta declarar a função e os valores iniciais. É possível também selecionar um ponto inicial com o mouse diretamente no gráfico. Também pode-se gerar arquivos em html para disponibilização na Internet.

Figura 7 - Exemplo do pacote animation para o método Newton-Raphson

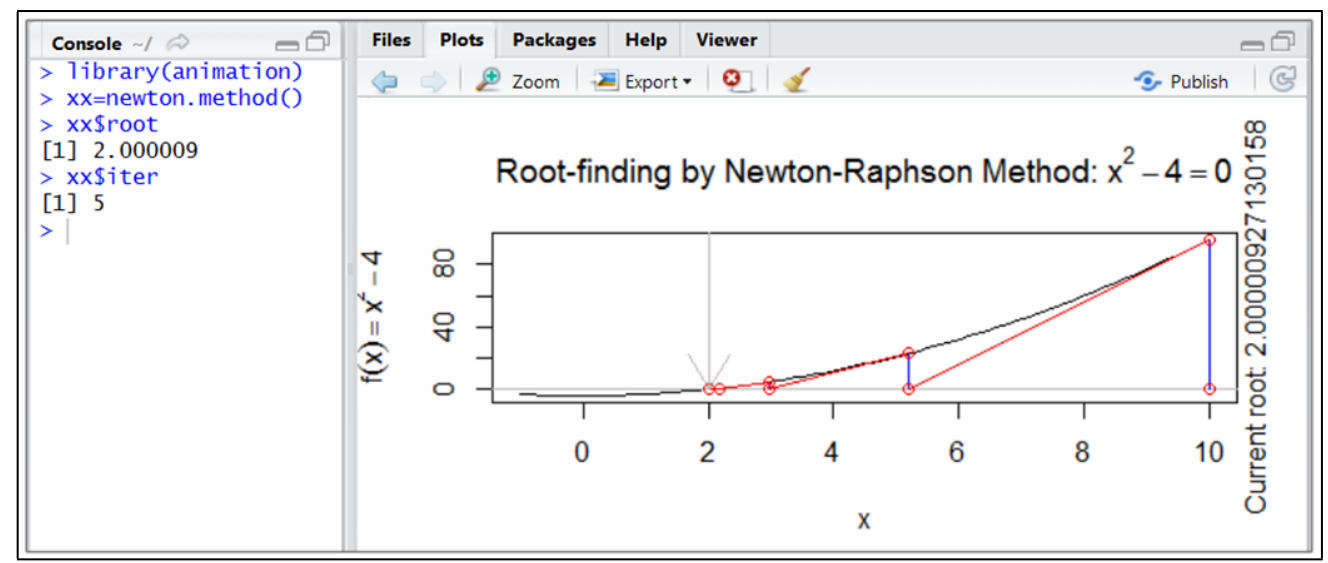

Fonte: produção dos autores 
Para finalizar, ressaltamos que a instalação padrão do $\mathrm{R}$ tem funções específicas para aproximar raízes de equações, mas optou-se, neste trabalho, pela abordagem de pacotes que possam auxiliar os estudantes na compreensão dos métodos estudados.

\section{Desenvolvimento de rotinas}

Apresentamos, em seguida, algumas rotinas elaboradas no $\mathrm{R}$ para os métodos do ponto fixo e Newton-Raphson. Nosso objetivo aqui é mostrar que a construção pode ser simples, partindo-se dos algoritmos estudados em aula. Assim, acreditamos que um estudante que cursa Cálculo Numérico possa elaborar programas e posteriormente aplicá-los.

Na Figura 08, apresentamos uma função para o método da secante desenvolvida no R, em um tablet (Sistema Android). A rotina é simples e a execução do código fornece uma raiz aproximada quando são declaradas a função, valores e tolerância. $A$ sintaxe utilizada serve para quaisquer outros sistemas.

A proposta é que os acadêmicos possam desenvolvê-las no próprio ambiente de aula, seja presencial ou a distância. Nesse sentido, notebooks, tablets e smartphones, aliados a pacotes livres, são alternativas que surgem como elementos construtores de novas práticas pedagógicas apoiadas na tecnologia. Para Dias e Araújo (2013) o uso de dispositivos móveis, tais como smartphones e tablets, apresenta um novo momento para as possibilidades de uso da tecnologia na educação.

\section{Figura 8 - Rotina implementada no sistema Android}

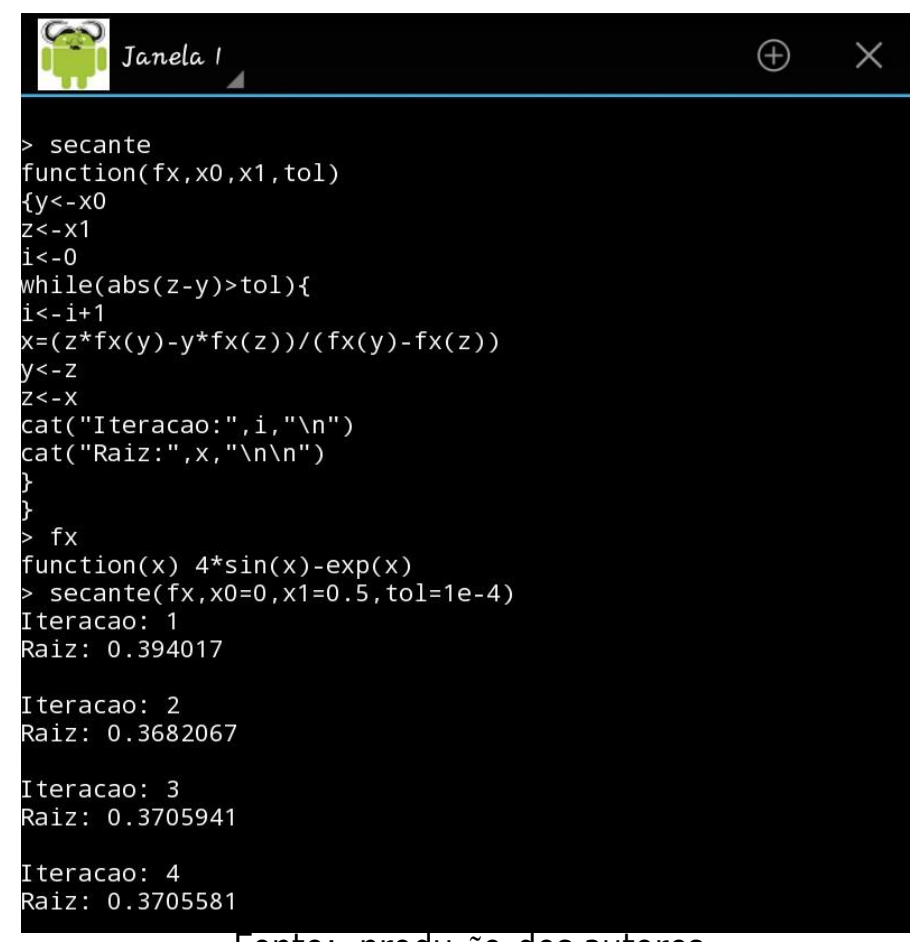

Fonte: produção dos autores

As tecnologias móveis permitem a ampliação do espaço e do tempo da aprendizagem e possibilitam dar suporte a estratégias de ensino e aprendizagem ativas, interativas e colaborativas. Seu uso adequado poderá promover a aprendizagem e as mudanças necessárias ao contexto educacional atual para a resolução ou a minimização de 
problemas nacionais como o ensino de Ciências, Tecnologia e Matemática (DIAS; ARAUJO, 2013).

Nossa proposta de incluir o $\mathrm{R}$ como opção no ensino de métodos numéricos vai ao encontro do exposto no trabalho de Cepeda (2013). Para o autor, tecnologia serve para melhorar a aprendizagem e dar apoio aos estudantes, combinando o uso de computadores e recursos móveis. Esses últimos ampliam as ferramentas auxiliares para o estudo, flexibilizando o ensino dessa disciplina e permitem desenvolver novas habilidades. Ainda, de acordo com Cepeda (2013), ao longo da última década, a propagação de linguagens de programação, o uso de softwares e inclusão de aplicações reais permitiram avançar de um curso tradicional de métodos numéricos para uma disciplina integrada com simulação computacional. É possível, em virtude da flexibilização existente, dar ênfase na visualização e simulação preenchendo assim uma importante lacuna entre a teoria e a prática.

Nesse contexto, o professor pode instigar os alunos a implementarem rotinas de conteúdos específicos de cálculo numérico em ambientes tecnológicos, combinando a teoria com a prática. É uma maneira diferenciada de ensino, pois esses ambientes possibilitam criar inúmeras simulações, propiciando ao aluno estabelecer conjecturas, validar e/ou refutar hipóteses. Na rotina de implementação, o aluno precisa transitar entre a técnica e a teoria, o que Ihe dá a possibilidade de trilhar um caminho de construção de conhecimento de forma autônoma.

O fato de usar ambientes computacionais para realizar tais tarefas não significa abandonar a tecnologia do lápis e papel, mas sim que essas tecnologias podem se complementar e propiciar um ambiente no qual 0 aluno pode transitar entre os diversos tipos de representação de um mesmo objeto.

Um exemplo de atividade que pode ser realizada envolve a utilização dos métodos numéricos da bissecção, de Newton-Raphson, do ponto fixo e da secante para determinar os zeros de uma função e, por meio dos resultados dados pelo $\mathrm{R}$ comparar número de iterações, precisão e a solução. Por exemplo, a função $f(x)=e^{-x^{2}}-\operatorname{cosx}$, explorando a velocidade de convergência em cada um dos métodos aplicados.

Outra atividade é propiciar ao aluno a verificação de que um determinado método pode não ser adequado dependendo do tipo de função dada. Exemplificando, para a função $\mathrm{f}(\mathrm{x})=\mathrm{x}^{3}-2 \mathrm{x}+2$, com $\mathrm{x}_{0}=0$, o método de Newton-Raphson não convergirá para a solução, pois fornecerá a seguinte sequência de termos $\{0,1,0,1,0,1,0,1,0,1,0,1 \ldots \ldots\}$. Outros métodos seriam mais indicados, como por exemplo o método da bissecção. No caso da função $\mathrm{f}(\mathrm{x})=|\mathrm{x}|-\mathrm{e}^{\mathrm{x}}$, a limitação ocorre no método de Newton-Raphson (dependendo da condição inicial), pois a função não é diferenciável em zero.

Assim, muito mais que apenas resolver numericamente uma atividade, deve-se propiciar ao aluno a oportunidade de tomar decisões pautados pela teoria, questionando: "qual o método é o mais adequado?"; "existem diferentes métodos que resolvem uma mesma situação problema?; "quais as vantagens e limitações que cada método apresenta? " Para isso, é importante a proposição de atividades que levem o aluno a refletir sobre tais questões e interpretar as soluções apresentadas

\section{Considerações finais}

Esse trabalho teve como objetivo principal mostrar algumas potencialidades do pacote GNU R como ferramenta auxiliar para o ensino de métodos numéricos em cursos de graduação e de pós-graduação. Foi uma pesquisa exploratória e concluiu-se que o R é uma alternativa viável, importante e com flexibilidade quanto a sua utilização. 
A partir dos pacotes que o $\mathrm{R}$ disponibiliza, o estudante tem acesso a uma maior gama de rotinas, programas e animações que podem auxiliar no processo de compreensão desses métodos. $\mathrm{O}$ uso do $\mathrm{R}$ pode contribuir para tornar a aula mais atrativa e os processos de ensino e aprendizagem mais efetivos.

Para fi nalizar, gostaríamos de deixar algumas recomendações que poderão servir para a continuidade dos trabalhos. A exploração da potencialidade do pacote GNU R, apresentada neste trabalho, contemplou parte do conteúdo de zeros de funções. Assim, é interessante estender a investigação para os demais métodos contemplados pela disciplina e, também, aplicar e avaliar a utilização do R em turmas de Cálculo Numérico para, assim, analisar formas de utilização da tecnologia neste contexto.

\section{Referências}

AMARAL T. R.; LEITE N. M. G.; SILVA, A.O. O Ensino de Cálculo Numérico Utilizando o Scilab. In: VI Congresso Internacional de Ensino da Matemática, 2013, Canoas.

BORCHERS, H. W. pracma: Practical Numerical Math Functions. R package version 1.8.3, 2015. URL: <http://CRAN.R-project. org/package=pracma>.

BORBA M. C.; PENTEADO, M. G. Informática e Educação Matemática. 3a ed. Belo Horizonte: Autêntica, 2003.99p

BURDEN, R.; FAIRES, J. Análise Numérica. 8a Edição, São Paulo: Cengage Learning, 2008.

CEPEDA, F.J.D. A Numerical Methods Course Based on B-Learning: Integrated Learning Design and Follow Up. International Journal of Mobile and Blended Learning, v.5, n.1, p. 39-57, 2013.

DENARDI, R. M.; DIMURO, G. P. A.; FAGUNDES, A. LESSA, M. A. F. Laboratório Computacional de Física: uma Experiência de Ensino Integrado de Matemática Numérica e Física em Engenharia. In: Congresso Nacional de Matemática Aplicada e Computacional, 2001, Belo Horizonte. XXIV CNMAC, Belo Horizonte: Gráfica Novo Milênio Ltda, 2001, v. 1, p. 1-316.

Dias, E.; Araujo Jr., C.F. Mobile learning no ensino de Matemática: um framework conceitual para uso dos tablets na Educação Básica. Encontro de Produção Discente PUCSP/Cruzeiro do Sul, v.1, n.1, p. 1-13, 2012.

FERREIRA, D.S; CYMROT, R. Uso do software R no tratamento estatístico de dados na Engenharia. In: Congresso de Iniciação Científica do Inatel - Incitel, 2012, Minas Gerais. Anais... São Paulo: Universidade Presbiteriana Mackenzie, 2012.p. 251257.

FOX, J. The R Commander: A Basic-Statistics Graphical User Interface to R. Journal of Statistical Software, v. 14, n. 9, p. 1-42, 2005.

OWEN, J. et al. spuRs: Functions and Datasets for "Introduction to Scientific $\mathrm{c}$ Programming and Simulation Using R". R package version 2.0.0, 2014. URL: $<$ http://CRAN.R-project.org/ package=spuRs $>$. 
R CORE TEAM. R: A language and environment for statistical computing. $R$ Foundation for Statistical Computing, Vienna, Austria, 2015, url http://www.Rproject.org/

RACINE, J. S. RStudio: A Platform-Independent IDE for R and Sweave. J. Applied Econometrics, v.27, p. 167-172, 2012.

RAMOS, D. M. C.; ARAUJO, W. B.; OLIVEIRA, A. R. Aplicação de métodos numéricos através ambiente gráfi co no ensino de engenharia. In: XXVIII Encontro Nacional de Engenharia de Produção, 2008, Rio de Janeiro.

TALES OF $R$. Install $R$ in Android, via GNURoot -no root required!. URL: $<$ https://talesofr.wordpress.com/tag/ r-console-free/>.

Running $\mathrm{R}$ on an iPhone/iPad with RStudio. URL: https://statisfaction.wordpress.com/2011/05/03/running-r-on-an-iphoneipad-withrstudio/

SOETAERT, K. rootSolve: Nonlinear root fi nding, equilibrium and steady-state analysis of ordinary diff erential equations. $R$ - package version 1.6, 2009. URL: $<$ http://CRAN.R-project.org/ package=rootSolve $>$.

XIE, Y. animation: An R Package for Creating Animations and Demonstrating Statistical Methods. Journal of Statistical Software, v.53, n.1, p. 1-27, 2013. 